\title{
Interface model of low temperature plasticity in high uniaxially strained monocrystalline semiconductors
}

\author{
Ye.F. Venger, V.V. Kolomoets, V.F. Machulin \\ Institute of Semiconductor Physics, NAS of Ukraine, 45, prospect Nauki, 03028 Kyiv, Ukraine
}

\begin{abstract}
The manifestation of the low temperature plasticity (LTP) in highly uniaxially strained $\mathrm{Ge}$ and $\mathrm{Si}$ single crystals was deduced from analysis of the both tensoeffect measurements data and defect-selective etching patterns of specimens. An appearance of additional tensoeffect mechanisms after the LTP display we attribute to the generation of electrical active defects of crystalline structure when the applied stress exceed some critical one. We found that under LTP conditions the generated dislocation pile-ups are directly concentrated in the phase-boundary field of some structural imperfections of crystalline lattice. The interface model of LTP phenomenon in monocrystalline semiconductors was proposed for acceptable explanation of the dislocation generation in the initially dislocation-free crystals.
\end{abstract}

Keywords: semiconductors, interface, uniaxial, deformation, dislocation, defect-selective-etching. Paper received 26.04.00; revised manuscript received 27.06.00; accepted for publication 30.06.00.

\section{Introduction}

The high temperature plasticity (HTP) of covalent crystals which takes place approximately at $T>T_{D}$ has been successfully explained within the model of motion and multiplication of dislocations [1]. The low temperature plasticity of a crystal with the large Peierls barrier should not be caused by the same mechanism as the HTP, since mobility of dislocations in semiconductors is low at $T<300 \mathrm{~K}$ [2]. Therefore, one could suppose that because of fresh dislocations generation under the LTP mechanism the dislocations' pileups will be localized in the region of the interface which separates different crystalline phases. Moreover, due to presence of the Cottrell atmosphere [3] on as-grown dislocations, an influence of electrically active defects on the electrophysical properties becomes visible only at substantial density of defect [4]. Vacant bonds of fresh dislocations, which are generated in highly uniaxially strained semiconductors at low temperatures, should create corresponding energy levels in the forbidden band. Thus, the influence of the LTP defect generation on electrophysical properties of semiconductors should be more significant as compared to the HTP case.

In this work, we demonstrate the data on the LTP displays in extremely highly strained $n-\mathrm{Si}(\mathrm{P})$ and $n-\mathrm{Ge}(\mathrm{Sb})$ crystals. The analysis of the non-trivial behavior of the transport phenomena in extremely strained $n$-Si and $n$-Ge crystals, which could not be explained by tensoeffect mechanisms known for Hookean strain region, was used for observation of LTP displays in monocrystalline semiconductors. We show that study of appropriate change of transport phenomena under high uniaxial pressure is a suitable method to investigate electrically active defect generation caused by the LTP in semiconductors. A defect-selective-etching method was used for confirmation of dislocation generation in highly strained crystalline semiconductors at $T \leq 300 \mathrm{~K}$ as well.

\section{Experimental}

The samples studied here were taken from sufficiently pure $n$-Si and $n$-Ge crystals doped in the melt with phosphorus and antimony, respectively. Such specimen selection allows us to realize the dominant tensoeffect mechanism of carriers redistribution between the $D_{1}$-minima in $n$-Si and the $L_{1}$-minima in n-Ge in the Hookean elasticity region [5]. Moreover, a low concentration of donors in $n-$ $\mathrm{Si}$ and $\mathrm{n}-\mathrm{Ge}$ ensures the necessary sensitivity of effects measured to the influence of the LTP defect generation. Measurements were carried out on the prism shape specimens with the two pairs of potential contacts hooked up to two $x$-y-recorders. The dimensions of the specimens are $\approx(0.8 \times 0.8 \times 8) \mathrm{mm}^{3}$. Fabrication of electric contacts 


\section{E.F. Venger et al.: Interface model of low temperature plasticity in high unixially ...}

to the sample was described earlier repeatedly. The accuracy of the x-ray method of crystallographic orientation was $\pm 15^{\prime \prime}$. Disorientation of the applied stress with respect to the corresponding crystallographic axis was better than $\pm 30^{\prime}$. Classical chemical solution for defectselective etching was used to study the images of dislocation distribution. Experimental data on tensoresistivity of crystals were obtained by using of original computerized installation for investigation of physical properties of solids under high uniaxial pressure, an elementary version of which was described earlier [6].

\section{Results and discussion}

High pressure tensoresistivity (TR) data for $n-\mathrm{Si}(\mathrm{P})$ and $n-\mathrm{Ge}(\mathrm{Sb})$ crystals are shown in Figs 1(a) and 1(b), respectively. In order to identify the deviation from the TR mechanism caused by the electrons redistribution between the $D_{1}$-minima in n-Si and the $L_{1}$-minima in n-Ge, an approach of deformation potential constant $\Xi_{u}$ determination was used [7]. For nondegenerate distribution [8], the ratio of electron concentration in the upper valley $\left(n_{2}\right)$ to the one in the lower valley $\left(n_{1}\right)$ can be given by the relationship:

$$
\frac{n_{2}}{n_{1}}=\exp \left(-\frac{\delta \varepsilon}{k T}\right),
$$

where $\delta \varepsilon$ is the energy gap between valleys of the conduction band in uniaxially strained many-valley semicon-

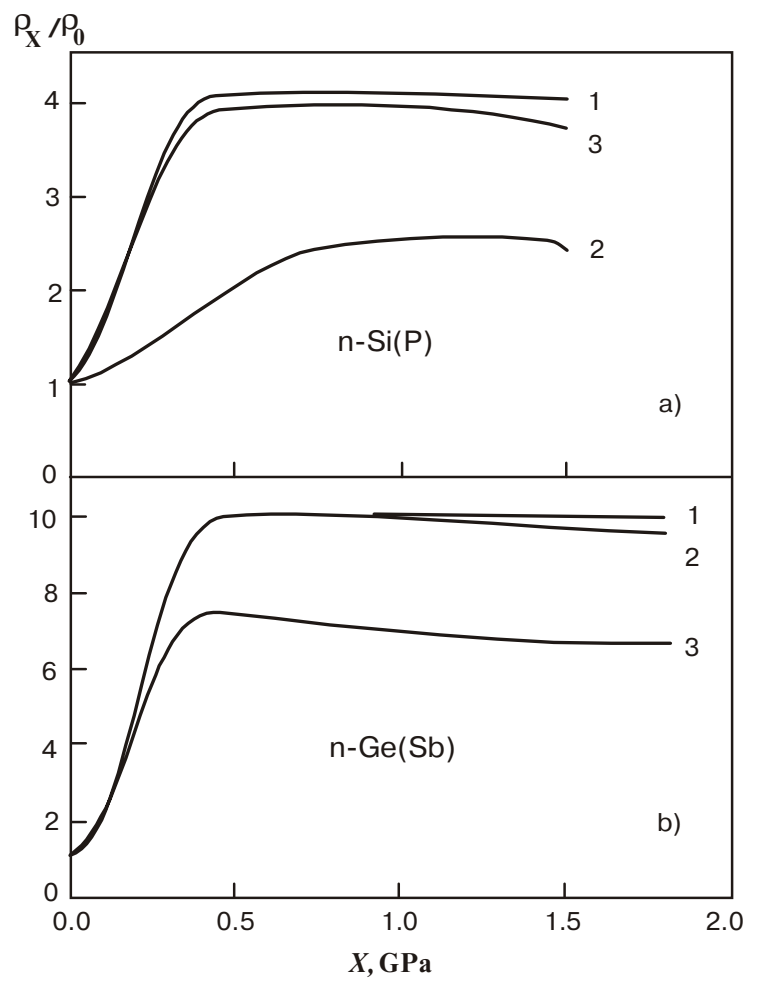

Fig. 1. Dependencies of $r_{X} / r_{0} v s \mathrm{X}$ for (a) $n$-Si(P) (X||[001]J) and (b) $n-\mathrm{Ge}(\mathrm{Sb})(\mathrm{X} \|[111] \mathrm{J})$. Recording sequence coincided with the curve number. ductors. In the cases related to our experimental condition the energy gap $\delta \varepsilon$ in the relation (1) is directly proportional to the pressure $X$ [9]:

de $\sim \Xi_{u} X$.

Therefore, for both the tensoresistivity mechanism of intervalley redistribution of electrons and $T=$ const condition equation (1) can be reduced to the following proportionality:

$\lg \left(n_{2} / n_{1}\right) \sim X$

The data on TR measurements obtained for a wide range of pressure allow to determine the ratio of electron concentrations determined by Eq. (1) for any magnitude of $X$ [10]. Following the method described in [10], we obtain corresponding relations for $n$-Si (under the condition $X||[001] \| J)$

$$
\frac{n_{2}}{n_{1}}=\left(1-\frac{\rho_{X}}{\rho_{\infty}}\right)\left(2 K \frac{\rho_{X}}{\rho_{\infty}}-2\right)^{-1}
$$

and for $n$-Ge (under the condition $X||[111] \| J$ )

$$
\frac{n_{2}}{n_{1}}=\left(\frac{\rho_{\infty}}{\rho_{X}}-1\right)\left(\frac{8 K+1}{3}-3 \frac{\rho_{\infty}}{\rho_{X}}\right)^{-1},
$$

where $K=\mu_{t} / \mu_{l}$ is a mobility anisotropy parameter. The magnitude of $K$ was determind for $n$-Si $(X \mid\|[001]\| J)$ and $n$-Ge $(X||[111] \| J)$ by the data on high pressure TR measurements as well [10]:

$K=\frac{3}{2} \frac{\rho_{\infty}}{\rho_{0}}-\frac{1}{2}$

The TR experimental data (Fig.1) processed by the presented approach of tensoeffect mechanism analysis are plotted in Fig. 2 (a, b) for $n$-Si and $n$-Ge, respectively. It is shown that for the cases of Hookean elasticity conditions, the proportionality of $\lg \left(n_{2} / n_{1}\right)$ on $X$ really takes place (curves 1, 3 in Fig 2 (a) and curves 1, 4 in Fig. 2 (b)). Non-linear and nonmonotonic dependencies of $\lg \left(n_{2} / n_{1}\right)=f(X)$ were obtained for $\mathrm{n}$-Si (curves 2,4 in Fig. 2 (a)) and $n$-Ge (curves 2, 3, 5 in Fig.2 (b)) after the LTP occurrence in extremely highly strained crystals.

Nonmonotonic behavior of $\lg \left(n_{2} / n_{1}\right)=f(X)$ dependencies in plastically deformed crystals can be explained by the generation of electrical active defects which are characterized both by the decrease and increase of an ionization energy in different pressure regions.

Generation of both single dislocations and pileups was confirmed by the defect-selective etching of specimens LTP detects of which are created when some limit of uniaxial pressure is exceeded. Defect-selective etching images of specimens after LTP occurrence, which was detected by the TR measurements (Fig. 1), and fol- 


\section{E.F. Venger et al.: Interface model of low temperature plasticity in high unixially ...}

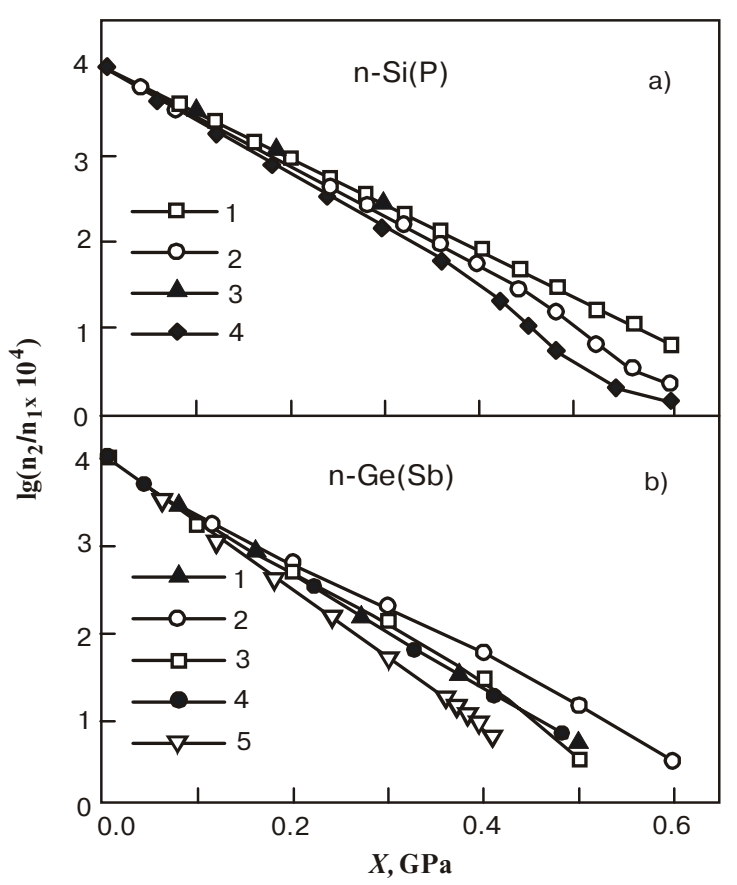

Fig. 2. Pressure dependencies at $T=78 \mathrm{~K}$ of $\lg \left(n_{2} / n_{1} \times 10^{4}\right)$ value for (a) $n-\mathrm{Si}(\mathrm{P})$ (curves 1,3 correspond to the elasticity condition, curves 2, 4 were obtained after the LTP manifestation) and (b) $n$-Ge(Sb) (curves 1, 4 for condition of elastic deformation, curves 2, 3, 5 for plastic deformed specimens. Curve 3 is an analogous dependence for specimen 2 after its annealing at $450 \mathrm{~K}$ for $1.5 \mathrm{~h}$ ).

lowing annealing of germanium crystals are presented in Fig. 3. It is shown that pileups which are generated under the LTP condition were formed either directly nearby the faces of mechanical stress application (Fig. 3a) or in the various interfaces regions, for example on the reconstructed crystal surfaces (Fig. 3b) or around the im- purity cluster embedded into the crystalline lattice, Fig. 3c), etc. Thus, the low mobility of dislocations generated in semiconductors at $T<300 \mathrm{~K}$ determines their localization in the region of interfaces of structural imperfections in crystal. This phenomenon could be used for crystalline structure perfection testing.

\section{Conclusions}

Low-temperature plasticity phenomenon in highly uniaxially strained $\mathrm{Si}$ and $\mathrm{Ge}$ was examined by means of tensoresistivity measurement and defect-selective etching methods. Interface model was proposed in order to supply an explanation of LTP phenomenon in highly strained monocrystalline semiconductors. Both the indirect experimental data and direct ones obtained under investigation of strain-induced phenomena verify proposed interface model of LTP. It was shown that the dislocations pile-ups which generated under LTP condition are localized directly in the range of different phase boundaries attributed to the some crystal lattice defects. The following type structural imperfections could belong to these kinds of defects, namely: inclusions of impurity atom aggregations, structural and nonstructural precipitates, embedded clusters of ensembles of impurity atoms and original lattice ones, extended defects on reconstructed crystal surfaces covered by either various chemical species or clusters, etc. So, given lattice mismatched interfaces on the various defect phase boundaries in the both crystal volume and essentially crystal surfaces and distinct response of the different crystal phases to the applied external stress may cause the dislocations generation in the interfaces region at once.

We demonstrate that the study of appropriate change of transport phenomena is a suitable method to investigate on electrical active defect generation caused by LTP effect as well.

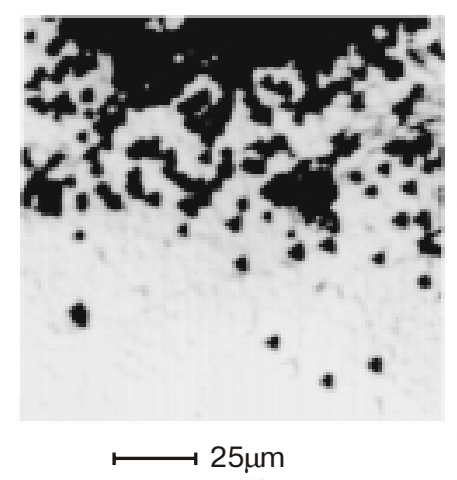

a)

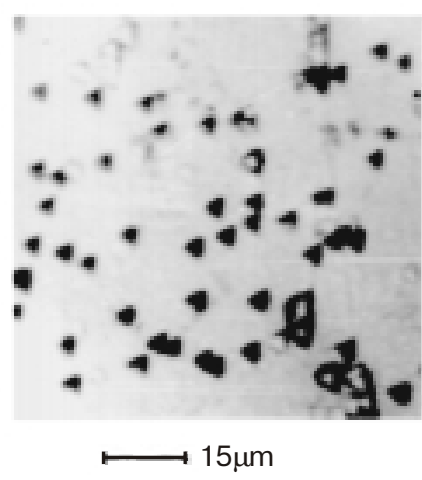

b)

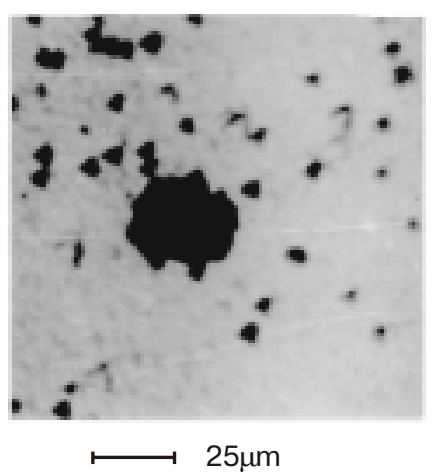

c)

Fig. 3. Metallographic images of the etched Ge crystals after both LTP display and specimes annealing $\left(T=850^{\circ} \mathrm{C}, t=2.5\right.$ hours) a) at the upper face of loaded specimen; b) on the reconstructed surface of specimen; c) around impurity cluster in crystal volume. 


\section{E.F. Venger et al.: Interface model of low temperature plasticity in high unixially}

\section{References}

1. G.L. Pearson, F.L.I. Vogel, Progress in Semiconductors, 6 (1962) 1.

2. K. Stokbro, L.B. Hansen, B.I. Lundqvist, in: Proc. of 23rd Int. Conf. on Phys. of Semicond. Berlin, (1996) v. 4, p. 2685.

3. A.H. Cottrell, Dislocations and Plastic Flow in Crystals (Claredon Press, Oxford, 1953) p.212.

4. H.F. Matare, Defect Electronics in Semiconductors. WileyInterscience a Division of John Wiley and Sons, Inc., New York-London-Sydney-Toronto, (1971) p. 458.

5. V.V. Baidakov, V.N. Ermakov, A.E. Gorin, V.V. Kolomoets and V.A. Shenderovskii // J. Phys. Chem. Solids, 56, 3/4 (1995) 319 .
6. V.V. Kolomoets and B.A. Suss, Soviet Phys. - Pribor. \& Tekhnika Exper, № 4 (1975) 214.

7. P.I. Baranskii, I.V. Dakhovskii, V.V. Kolomoets, A.V. Fedosov, Soviet Phys. - Fiz. i Tekhnik. Poluprovodn, 10, (1976) 1387.

8. J.S. Blakemore, Semiconductor Statistics (Pergamon Press, London, 1962) p. 381.

9. G.L. Bir and G.E. Pikus, Symmetry and Strain-Induced Effects in Semiconductors Wiley, New York, (1974) p. 538.

10. P.I. Baranskii, V.V. Kolomoets, A.V. Fedosov, V.P. Shapovalov, Soviet Phys. - Fiz. i Tekhnik. Poluprovodn, 15 (1981) 864. 